\title{
Evaluation of access to adrenaline in different non-operative clinical areas-A Blind Study
}

\author{
Dr.Haider Abbas, Dr.Nikhil Kothari, Dr.Qazi Ehsan Ali, Dr.M Parvez Khan, \\ Dr.Jaishree Bogra, Dr Syed Hussain Amir, Dr.Ahlam kazim \\ Associate Professor Department of Anaesthesiology K G Medical University Lucknow-226003India \\ Senior Resident Department of Anaesthesiology K G Medical UniversityLucknow-226003 India \\ Asociate Professor Department of Anaesthesiology J N Medical College AMU, Aligarh-202002 India \\ Professor Department of Anaesthesiology K G Medical University Lucknow-226003 India \\ Professor Department of Anaesthesiology K G Medical University Lucknow-226003 India \\ Assistant Professor Department of Anaesthesiology J N Medical College AMU, Aligarh-202002 India \\ Demonstartor Department of Biochemistry K G Medical University Lucknow-226003 India
}

\begin{abstract}
Introduction: The impulse for carrying out this study stemmed from the fact that there was an observational variation in responding to and conducting the management of critical incidents by residents (senior house officers) in different wards of the hospital. Following an incident where intravenous adrenaline was not available in adequate time during sudden cardiac arrest; the present study was done to objectively evaluate the access to the prototype emergency drug,adrenaline injection.Material and Method: Ten teams were sent to five different wards to simultaneously check the response of duty doctors to pick up Adrenaline injection in any form (pre-filled syringes or ampoules) at the nursing station and hand it over to the auditor. Results: The time to access the adrenaline in ampoule form was least in Accident \& Emergency $(24.33 \pm 3.67$ sec) unit while highest in Medicine ward $(32.17 \pm 3.66 \mathrm{sec})$. The time to access the adrenaline in pre-filled syringes was least in Accident \& Emergency (19.00 $\pm 2.71 \mathrm{sec})$ unit while highest in Obstetrics ward $(23.50 \pm$ $1.00 \mathrm{sec}$ ). Conclusion: Time to access adrenaline injection was shorter in the Accident \& Emergency unit but was significantly higher $(p<.05)$ in other wards; also the access to pre-filled syringes was quicker than the ampoules.
\end{abstract}

\section{Introduction}

Adrenaline is a vital emergency drug required in life threatening conditions.. It's vital to have quick access to adrenaline as delay in procurement of injection will have serious impact on the recovery in life threatening conditions. Access to such medicines can be compromised at times because healthcare professionals are normally very busy in the wards with lot of parallel processes going on in the general wards, lack of orientation plus the absence of crash trolleys in some of the hospitals, therefore the present study was done to evaluate the time to access the prototype emergency drug, adrenaline injection in different wards, which in turn is one of the very important factors for the survival of the patient.

\section{Material and methods}

The study was undertaken in five different non-operative clinical areas namely Accident and Emergency (A\&E), Medical and Surgical ward, Obstetrics, Trauma and Orthopaedics wards of the hospital. Ten resident doctors in each ward participated in the study. Inclusion criteria of our study were, only those resident doctors, who were not attending any patient at the time of study were included in our study. Teams were sent to five different wards to check the response of duty doctors. These teams comprising of two persons; one Resident Doctor, who acted as an auditor and other person, a technician; who kept the records and observed the Doctor being studied. After explaining them to pick up the adrenaline injection in any form (pre-filled syringes or ampoules) at the nursing station after the stop watch starts and hand it over to the auditor. Access time to get adrenaline ampoules/prefilled syringe in seconds was measured by stop watch.

\section{Statistical analysis}

Time (sec) to access adrenaline in five different non-operative wards (groups) were compared by one factor analysis of variance (ANOVA) while time to access two types (AMP and PFS) of adrenaline in five different groups were compared by two factor ANOVA and the significance of mean difference within and between groups were done by Newman-Keuls post hoc test. A two-tailed $(\alpha=2)$ probability $(p)$ value $p<0.05$ was considered to be statistically significant. STATISTICA (version 6.0) was used for the analysis 


\section{Result}

Time (sec) to access adrenaline in different non-operative wards is summarized in Table 1. Mean time to access adrenaline was least in A\&E ward $(22.20 \mathrm{sec})$ while highest in Medicine ward $(38.00 \mathrm{sec})$. The Medicine ward also showed the highest variability (21.6\%) in time to access the adrenaline while Obstetrics the least $(9.3 \%)$. On comparing the time, the mean time to access adrenaline in Medicine ward was found to be significantly $(p<0.05)$ higher than the A\&E while it did not differed $(p>0.05)$ in other wards.

The time (sec) to access adrenaline in five different non-operative wards was further studied according to its procurement either in Ampoule form (AMP) or Prefilled syringe form (PFS) and it wasfound that time to access the adrenaline in Ampoule form was least in A \& E $(24.33 \pm 3.67 \mathrm{sec})$ while highest in Medicine ward $(32.17 \pm 3.66 \mathrm{sec})$. Similarly, the time to access the adrenaline in Prefilled syringe form was least in A\&E $(19.00 \pm 2.71 \mathrm{sec})$ while highest in Obstetrics ward $(23.50 \pm 1.00 \mathrm{sec})$. It was also observed that mean time to access PFS were less than the mean time to access AMP in each group.

For each access, comparing the time between the wards (within groups), the mean time to access adrenaline in AMP form differed significantly $(\mathrm{p}<0.01)$ between A \& E and Medicine (Table 2). The mean time to access adrenaline in AMP form also differed significantly $(\mathrm{p}<0.05)$ in Surgery, T \& O and Obstetrics as compared to Medicine (Table 2). However, the mean time to access adrenaline in PFS form did not differed ( $p>0.05$ ) between the wards i.e. found to be statistically the same (Table 2).

Similarly, for each ward, comparing the time between two types (between groups), the mean time to access adrenaline in all wards differed significantly (either $\mathrm{p}<0.05$ or $\mathrm{p}<0.01$ ) between AMP and PFS except T $\& \mathrm{O}$ ward (Table 2). In $\mathrm{T} \& \mathrm{O}$ ward, the mean time to access adrenaline either in AMP form or PFS form was found to be the same (Table 2).

\section{Discussion}

Adrenaline is a neurotransmitter which effects heart rate, blood pressure, dilates air passages and thus can reverse haemodynamic compromise in certain life-threatening circumstances through its vasoconstricting, positive inotropic, bronchodilating properties and by reducing mast-cell and basophil mediator release. ${ }^{[1,2]}$

The commercially available auto-injector epinephrine is considerable expensive. Epinephrine prefilled syringe is an alternative for easy access during cardiac arrest emergency and for treatment of anaphylaxis patients. It was also observed that the response to pick up PFS form of adrenaline was less as these were stored in the pencil box to prevent from light exposure and were easier to locate. Consequently, epinephrine prefilled syringe was stable and sterile at least three month after preparation. ${ }^{[3]}$

In environments with airborne contaminants, the use of prefilled syringes may be useful for preventing bacterial contamination of the medicine inside, ${ }^{[4]}$ but need to be replaced after few months to maintain efficacy. ${ }^{[5]}$

Access to Adrenaline is very vital in some life-threatening scenarios, so to procure it at times of need is understandable. In cardiac arrest it is said that "time is tissue" and each minute delay in adrenaline injection can reduce the chances of survival. In cases of anaphylactic shock the earlier the administration of adrenaline will lead to quick haemodynamic control and less end organ damage. It should be administered as early as possible and titrated carefully to clinical response. Poor outcomes during anaphylaxis, including deaths are associated with delayed or no administration of epinephrine, as well as with inadequate or excessive dosing.

It can be seen from the studies that mean time of access to adrenaline injection varied from ward to ward in a single institute. Thus to have a mean time of aceess to adrenaline we require a much larger multicentric study using not only adrenaline but also many other such type of emergency drugs.

This study was first of its kind to be done. Such type of studies are required to be done more often to approximately calculate the mean time of access to various emergency drugs in required conditions .

\section{Conclusion}

We conclude that the time to access adrenaline injection was significantly shorter in the A\&E but it was significantly higher in other wards. The access to pre-filled syringes was quicker than the ampolues therefore it is advisable to place "adrenaline" in clearly demarcated area with colour coded illuminated signmarks and in preferably pre-filled syringes.

\section{References:}

[1]. Daul A, Hermes U, Schäfers RF, Wenzel R, von Birgelen C, Brodde OE.The beta- adrenoceptor subtype(s) mediating adrenalineand dobutamine-induced blood pressure and heart rate changes in healthy volunteers.Int J Clin Pharmacol Ther. 1995 Mar;33(3):1408 .

[2]. Adrenaline, cardiac arrest, and evidence based medicine T H Rainer and C E Robertso,J Accid Emerg Med. 1996 July; 13(4): 234-237

[3]. Soar J, Deakin CD, Nolan JP, et al. European Resuscitation Council guidelines for resuscitation 2005. Section 7. Cardiac arrest in special circumstances. Resuscitation 2005;67(Suppl 1):S135-70.

[4]. Kerddonfak S, Manuyakorn W, Kamchaisatian W, Sasisakulporn C, Teawsomboonkit W, Benjaponpitak S., The stability and sterility of epinephrine prefilled syringe,Asian Pac J Allergy Immunol. 2010 Mar;28(1):53-7. 
[5]. Ninomiya N, Koido Y, Yamamoto Y,Aseptic efficacy of prefilled syringes in a polluted environment Prehosp Disaster Med. 2001 JanMar;16(1):14

Table 1. Statistics of time (sec) to access adrenaline in different non-operative wards

\begin{tabular}{lccccc}
\hline Statistics & \multicolumn{4}{c}{ Wards } \\
\cline { 2 - 5 } & A \& E & Medicine & Surgery & T \& O & Obstetrics \\
\hline N & 10 & 10 & 10 & 10 & 10 \\
Mean & 22.20 & $28.00^{*}$ & 25.30 & 24.90 & 25.40 \\
SD & 4.18 & 6.06 & 4.40 & 3.35 & 2.37 \\
SE & 1.32 & 1.91 & 1.39 & 1.06 & 0.75 \\
Cov $(\%)$ & 18.8 & 21.6 & 17.4 & 13.4 & 9.3 \\
Min & 15 & 21 & 20 & 20 & 23 \\
Max & 29 & 38 & 34 & 29 & 30 \\
\hline
\end{tabular}

" $\mathrm{p}<0.05$ - as compared to A \& E

$\mathrm{N}=$ No. of resident physician participating in study

A \& E: Accident \& Emergency, T \& O: Trauma \& Orthopaedics, Cov- Coefficient of Variation

Table 2. Summary (Mean \pm SD) of time (sec) to access adrenaline in AMP and PFS forms in different non-

\begin{tabular}{|c|c|c|c|}
\hline \multirow[t]{2}{*}{ Wards } & \multicolumn{2}{|c|}{ Type of access } & \multirow{2}{*}{$\begin{array}{c}\mathrm{p} \text { value } \\
\text { (AMP vs. } \\
\text { PFS) }\end{array}$} \\
\hline & $\begin{array}{l}\text { AMP } \\
(n=6)\end{array}$ & $\begin{array}{c}\text { PFS } \\
(\mathrm{n}=4)\end{array}$ & \\
\hline$A \& E$ & $24.33 \pm 3.67$ & $19.00 \pm 2.71$ & 0.040 \\
\hline Medicine & $32.17 \pm 3.66^{\mathrm{a}}$ & $21.75 \pm 0.96$ & 0.000 \\
\hline Surgery & $27.83 \pm 3.82^{b}$ & $21.50 \pm 1.29$ & 0.012 \\
\hline $\mathrm{T} \& \mathrm{O}$ & $27.17 \pm 1.94^{\mathrm{b}}$ & $21.50 \pm 1.29$ & 0.033 \\
\hline Obstetrics & $26.67 \pm 2.16^{\mathrm{b}}$ & $23.50 \pm 1.00$ & 0.172 \\
\hline
\end{tabular}

${ }^{\mathrm{a}} \mathrm{p}<0.01-$ as compared to A \& E

${ }_{\mathrm{p}}^{\mathrm{p}}<0.05$ - as compared to Medicine

AMP: Ampoules, PFS: Prefilled syringe, A \& E: Accident \& Emergency, T \& O: Trauma \& Orthopaedics 